\title{
BMJ Open Decomposition of socioeconomic inequalities in child vaccination in Ethiopia: results from the 2011 and 2016 demographic and health surveys
}

Firew Tekle Bobo (D) , 1,2 Andrew Hayen ${ }^{2}$

To cite: Bobo FT, Hayen A. Decomposition of socioeconomic inequalities in child vaccination in Ethiopia: results from the 2011 and 2016 demographic and health surveys. BMJ Open 2020;10:e039617. doi:10.1136/ bmjopen-2020-039617

- Prepublication history for this paper is available online. To view these files, please visit the journal online (http://dx.doi. org/10.1136/bmjopen-2020039617).

Received 22 April 2020 Revised 19 August 2020 Accepted 24 August 2020
D) Check for updates

(C) Author(s) (or their employer(s)) 2020. Re-use permitted under CC BY-NC. No commercial re-use. See rights and permissions. Published by BMJ.

${ }^{1}$ Department of Public Health, Institute of Health Sciences, Wollega University, Nekemte, Ethiopia

${ }^{2}$ School of Public Health, Faculty of Health, University of Technology Sydney, Sydney, New South Wales, Australia

Correspondence to

Firew Tekle Bobo;

free11messi@gmail.com

\section{ABSTRACT}

Objectives Monitoring and addressing unnecessary and avoidable differences in child vaccination is a critical global concern. This study aimed to assess socioeconomic inequalities in basic vaccination coverage among children aged 12-23 months in Ethiopia.

Design, setting and participants Secondary analyses of cross-sectional data from the two most recent (2011 and 2016) Ethiopia Demographic and Health Surveys were performed. This analysis included 1930 mother-child pairs in 2011 and 2004 mother-child pairs in 2016.

Outcome measures Completion of basic vaccinations was defined based on whether a child received a single dose of Bacille Calmette-Guerin (BCG), three doses of diphtheria, tetanus toxoids and pertussis (DTP), three doses of oral polio vaccine and one dose of measles vaccine.

Methods The concentration Curve and Concentration Indices (CCls) were used to estimate wealth related to inequalities. The concentration indices were also decomposed to examine the contributing factors to socioeconomic inequalities in childhood vaccination. Results From 2011 to 2016, the proportion of children who received basic vaccination increased from $24.6 \%$ (95\% Cl $21.4 \%$ to $28.0 \%$ ) to $38.6 \%$ (95\% Cl $34.6 \%$ to $42.9 \%$ ). While coverage of BCG, DTP and polio immunisation increased during the study period, the uptake of measles vaccine decreased. The positive concentration index shows that basic vaccination coverage was pro-rich ( $\mathrm{Cl}=0.212$ in 2011 and $\mathrm{CCl}=0.172$ in 2016). The decomposition analysis shows that use of maternal health services such as family planning and antenatal care, socioeconomic status, exposure to media, urbanrural residence and maternal education explain inequalities in basic vaccination coverage in Ethiopia.

Conclusions Childhood vaccination coverage was low in Ethiopia. Vaccination was less likely in poorer than in richer households. Addressing wealth inequalities, enhancing education and improving maternal health service coverage will reduce socioeconomic inequalities in basic vaccination uptake in Ethiopia.

\section{INTRODUCTION}

Vaccination is an important public health intervention that helps prevent 2-3 million child deaths each year. ${ }^{1}$ With improved

\section{Strengths and limitations of this study}

- We analysed two most recent (2011 and 2016) nationally representative Demographic and Health Surveys (DHS).

- The decomposition of the contributing factors that drive socioeconomic inequalities in vaccination coverage provided a rich set of analysis for policy interventions to address socioeconomic disparities in child vaccination in Ethiopia.

- Limitations of the current study may include recall bias related to vaccination status as not all children had vaccination cards, and measures had to depend on the mother's verbal report.

- The DHS is a cross-sectional survey; it was not possible to establish temporality between childhood vaccination and explanatory factors, precluding causal inference.

coverage, vaccines have the potential to save many more children, which is why it is necessary to ensure that all children receive all recommended vaccines. ${ }^{23}$

In Ethiopia, a child is said to have received full vaccinations if they receive one dose of the Bacille Calmette-Guérin vaccine (BCG, for tuberculosis), three doses of the pentavalent vaccine (penta includes diphtheria, tetanus, pertussis, hepatitis B virus and haemophilus influenzae type b), three doses of the oral polio vaccine (OPV), three doses of the pneumococcal conjugate vaccine and two doses of the rotavirus vaccine (rota), and one dose of measles-containing vaccine. ${ }^{45}$ Basic vaccination is defined as access to a single dose of BCG, three doses of DTP, three doses of OPV and one dose of measles vaccine by the age of 12 months. $^{4}$

In the past decades, global basic vaccination coverage has improved remarkably. ${ }^{67}$ In 2018 , the proportion of the world's children who received three doses of the combined diphtheria, tetanus toxoid and pertussiscontaining vaccine (DTP3) reached $86 \%$ 
worldwide. ${ }^{1}$ However, there are inequalities in access to childhood vaccination and many children do not receive the basic vaccines worldwide. ${ }^{89}$

Many regions of the world continue to have low coverage. WHO report in 2019 shows that 19.4 million children under the age of 1 year did not receive basic vaccines; around $60 \%$ of these children live in 10 nations, including Nigeria, the Democratic Republic of the Congo and Ethiopia. ${ }^{1}$ Disparities in vaccination coverage exist within and between countries, and in some places; the difference is more significant. ${ }^{8}$ Within countries, inequalities in child vaccination data show that richer subgroups tend to have higher coverage whereas the coverage among poorer subgroups varies across countries. For example, studies in India, ${ }^{10}$ Nigeria $^{11}$ and Brazil ${ }^{12}$ indicated that children of mothers who had higher education levels and household wealth status are more likely receive higher vaccination coverage.

Complete lack or incomplete childhood vaccination remains the cause of millions of preventable child deaths each year in many countries. ${ }^{1}$ Previous studies in the area suggest that there exists a social gradient in child vaccination within countries. ${ }^{13-15}$ For example, increased vaccination coverage was favourably concentrated among children whose parents are well educated, wealthy or living in urban areas. Inequalities in access to child vaccination need to be effectively assessed, monitored and intervened to address systematically missed population groups. ${ }^{16} 17$ In 2019 , only $43 \%$ of children received all recommended vaccines while $19 \%$ received none of the vaccines. ${ }^{18}$ This is way below WHO's 2020 goal of $90 \%$ coverage in every country. ${ }^{19}$ Addressing these gaps requires measuring inequalities in basic vaccination coverage and identifying where gaps exist in routinely delivered vaccines and provide valuable information to introduce effective strategies and policies to address such inequalities. It is equally important those children who receive incomplete or no vaccines be identified to devise equity-oriented immunisation programmes to reach disadvantaged populations and reduce Ethiopia's high levels of vaccine preventable childhood morbidity and mortality.

Although there are previous studies ${ }^{20-22}$ in Ethiopia that have addressed factors associated with childhood vaccination, there is a need to examine trends and socioeconomic inequalities in childhood vaccination. The objective of this study is to examine trends and socioeconomic inequalities in childhood vaccination. Moreover, the paper assesses factors that explain socioeconomic inequalities in childhood vaccination in the country using a decomposition approach.

\section{METHODS}

\section{Data}

We analysed the most recent (2011 and 2016) Ethiopia Demographic and Health Surveys (DHSs). The Ethiopian Central Statistical Agency undertook the surveys in collaboration with the DHS programme. ${ }^{23}$ The DHSs are
Table 1 Basic vaccination schedule for children under 12 months in Ethiopia

\begin{tabular}{lll}
\hline Vaccine & Diseases & Age \\
\hline BCG & Tuberculosis & At birth \\
DTP & $\begin{array}{l}\text { Diphtheria, Pertussis, } \\
\text { Tetanus }\end{array}$ & $6,10,14$ weeks \\
OPV & Polio & At birth, 6, 10, 14 weeks \\
Measles & Measles & 9 months \\
\hline
\end{tabular}

BCG, Bacille Calmette-Guerin; OPV, oral polio vaccine.

nationally representative household surveys with large sample sizes and high response rates. ${ }^{24}$ The DHS uses a stratified, two-stage sampling technique to obtain the study participants. ${ }^{25}$ Standardised questionnaires are used across time and countries to ensure collected data are comparable. ${ }^{26}$ Sampling methods and design have been described elsewhere. ${ }^{27}$ For the purpose of this study, data collected on vaccination status of children aged 12-23 months were extracted and analysed. The Ethiopia DHS included information on 11872 births/women in 2011 and 11023 births/women in 2016. The sample used for the current analysis was limited to children aged 12-23 months at the time of the survey, yielding a final sample of 1930 mother-child pairs in 2011, and 2004 motherchild pairs in 2016.

\section{Measures}

The dependent variable is whether a child received all basic vaccinations that is the eight recommended basic vaccines. ${ }^{28}$ The vaccines included one dose of BCG against tuberculosis, three doses of DTP, three doses of OPV and one dose of measles vaccine. Table 1 shows immunisation schedule for children under 12 months in Ethiopia. ${ }^{4}$ The DHS determined the vaccination status of children from two sources. Primarily immunisation record cards provided by mothers, but if these were absent the DHS data collectors used mothers' verbal reports of children's immunisation status.

WHO Commission on Social Determinants of Health framework $^{29}$ was used to explain contributing factors of inequalities in vaccination status. In addition, factors identified in the current literature $\mathrm{e}^{1113143031}$ on child vaccination that are available in the DHS surveys were included. The independent variables considered in the current study include (1) maternal and household factors: maternal parity, age, education levels, wealth status and the use of maternal health services, (2) exposure to media, and (3) place of residence-urban/rural status.

The wealth index is a composite variable that measures the woman's household living standards. It is constructed by collecting and analysing information on ownership of selected materials and assets, such as radio, television (TV), refrigerator and vehicle; materials used for housing construction; and types of sanitation facilities and water 
access. Households were ranked into five quintiles (poorest, poorer, middle, richer and richest) depending on their level of wealth.

We grouped education levels of the mothers in to three categories (no education, primary and secondary or higher). Exposure to media: frequency of listening to radio and watching TV: both categorised as (not at all, less than once a week and once a week or more). Utilisation of reproductive and maternal health services considered in the current study include use of contraceptive, antenatal care contacts and delivery at the health facility.

\section{Statistical analysis}

Socioeconomic inequalities in vaccination coverage were estimated using the Concentration Curve and Concentration Index (CCI) ${ }^{32}$ The concentration curve is a plot of the cumulative percentage of the population, ranked by wealth status, from the poorest to the richest (x-axis) against the cumulative percentage of the health variable (vaccination status) on the y-axis. If all children had an equal proportion of vaccination status regardless of their socioeconomic status, then the curve would coincide with the $45^{\circ}$ line, which indicates the presence of equality in the coverage of vaccination. If the concentration curve falls below the $45^{\circ}$ line of equality, it indicates that the uptake of vaccines is more concentrated among the rich. The opposite is true if the curve falls above the line of equality.

The concentration index is described as two times the area between the line of equality and the concentration curve. The index takes a value between -1 and +1 ; an index of 0 indicates the presence of equality in the uptake of vaccines. If wealth-related inequalities exist, it can be seen in one of the two forms, the first is when there is uneven concentration of vaccine uptake among the rich, and in this case, the concentration index takes on a positive value. The second is negative value concentration index, which implies high concentration of vaccination status among the poor.

The concentration index (CCI) can be computed as follows:

$$
\mathrm{CCI}=\frac{2}{\mathrm{y}} \operatorname{cov}(\mathrm{h}, \mathrm{r}),
$$

where $\mathrm{h}$ is the healthcare outcome of interest (ie, vaccination status), $y$ is the mean of $h$ and $r$ is the fractional rank of an individual in the wealth distribution. We also computed 95\% CIs for the concentration index.

\section{Decomposing inequality}

The concentration CCI can only show and quantify the level of inequalities related to wealth in the use of health services. However, policy-makers are also interested in the factors that contribute to socioeconomic inequalities in vaccination coverage. This can be done using an approach developed by Wagstaff et al. ${ }^{33}$ The concentration index of a health variable can be decomposed into the contributions of individual factors to wealth-related health inequality. If we consider a linear regression model for the child's vaccination status, $v$, is defined according to $k$ explanatory factors, $x_{k}$ as.

$$
v=\alpha+\sum_{k} \beta_{k} x_{k}+\varepsilon,
$$

where $\alpha$ and $\beta$ are parameters, and $\varepsilon$ is the error term. The concentration index for child vaccination status can be decomposed as:

$$
C=\sum_{k}\left(\frac{\beta_{k} \bar{x}_{k}}{\mu}\right) C_{k}+\frac{G C_{\varepsilon}}{\mu},
$$

where $\mu$ is the mean of $y, \bar{x}_{k}$ is the mean of $x_{k}, C_{k}$ is the concentration index for $x_{k}$, (defined analogously to C), and $G C_{\varepsilon}$ is the generalised concentration index for the error term $(\varepsilon)$. Equation 3 shows that $C$ is equal to a weighted sum of the concentration indices of the $k$ regressors, where the weight for $x_{k}$ is the elasticity of $y$ with respect to $x_{k}\left(\eta_{k}=\beta_{k} \frac{\bar{x}_{k}}{\mu}\right)$. The residual component captured by the last term $\left(\frac{G C_{\varepsilon}}{\mu}\right)$ reflects the wealth-related inequality in health that is not explained by systematic variation in the regressors. We used the bootstrap method with 1000 replications to estimate standard errors . All analyses were performed after adjusting for sampling design (stratification and clustering) and sampling weights. STATA (V.14, StataCorp) and SPSS (V.26) software packages were used to perform data analysis.

\section{Patient and public involvement}

Patients/public were not involved in the design or implementation of this study.

\section{RESULTS}

The sample used for the current analysis was limited to children aged 12-23 months at the time of the survey, yielding a final sample of 1930 mother-child pairs in 2011, and 2004 mother-child pairs in 2016. The majority $(85.8 \%$ and $88.4 \%)$ of respondents were from rural areas in 2011 and 2016 surveys, respectively, and more than $42 \%$ were from Oromia, which is the biggest region in the country. The percentage of mothers who had no education decreased from $68 \%$ in 2011 to $64 \%$ in 2016 while antenatal care contacts increased from $42 \%$ in 2011 to $60 \%$ in 2016 (table 2).

\section{Trends of vaccination coverage}

Vaccination coverage showed improvements from 2011 to 2016; BCG vaccine uptake increased from $66 \%$ to $69 \%$, DTP3 vaccine from $37 \%$ to $57 \%$, OPV3 vaccine from $45 \%$ to $57 \%$, and basic vaccination coverage from $24 \%$ in 2011 to $38 \%$ in 2016 . The proportion of children who received all basic vaccinations that include BCG, DTP3, OPV3 and measles increased by $14 \%$ from 2011 to 2016 . However, measles vaccine coverage decreased from $56 \%$ in 2011 to $54 \%$ in 2016 , while the proportion of children who received no vaccination increased from $14 \%$ in 2011 to $16 \%$ in 2016 (figure 1). 


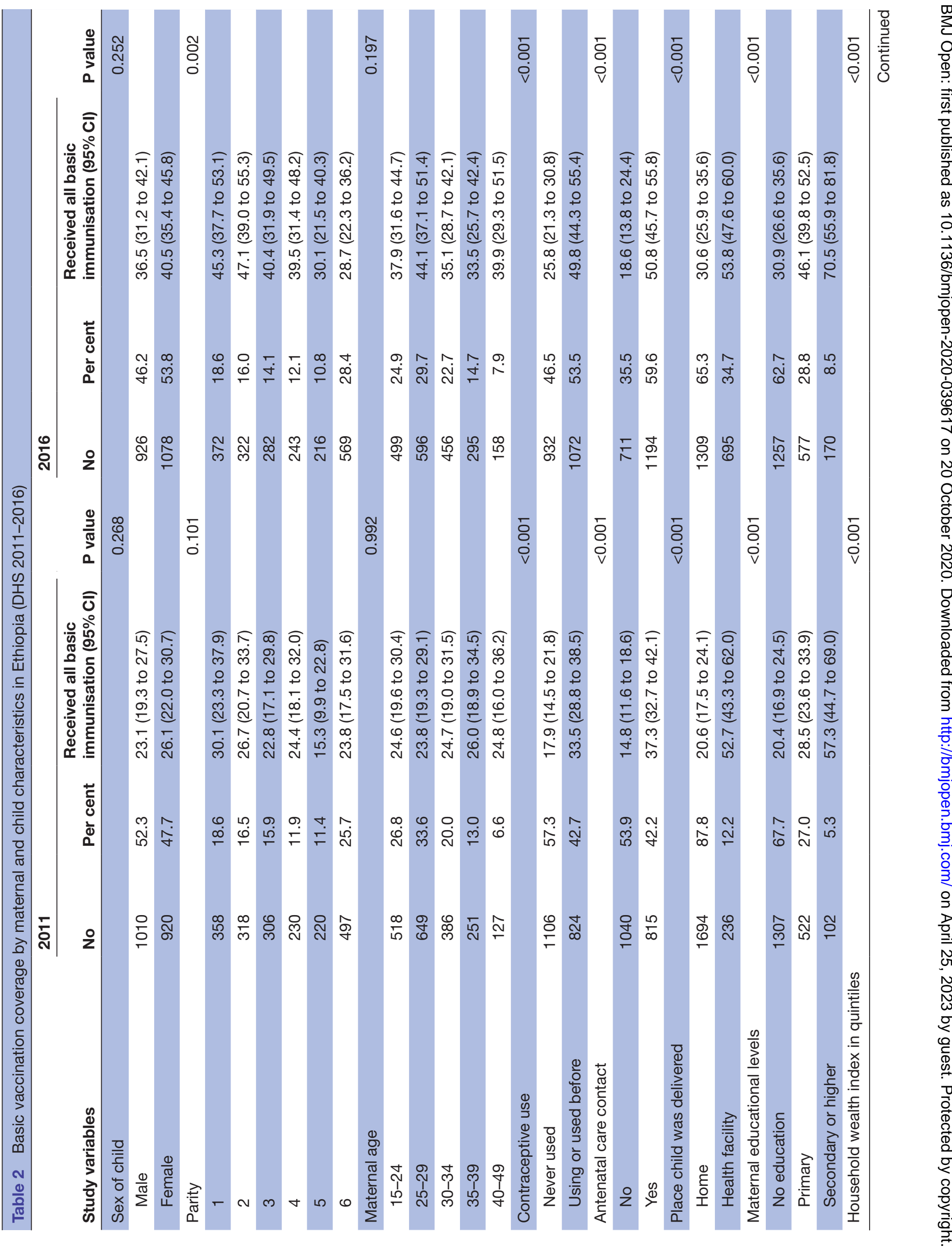




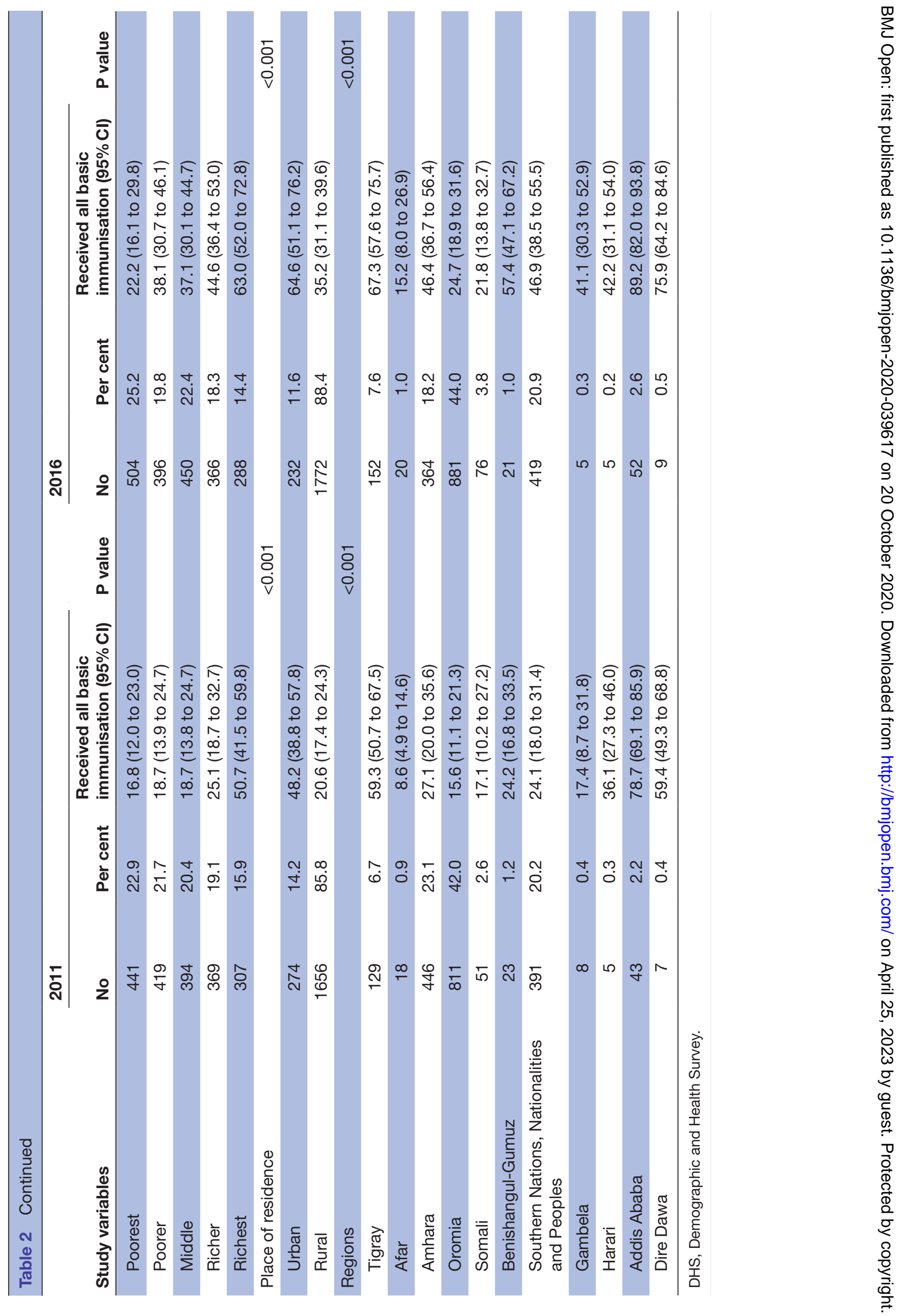




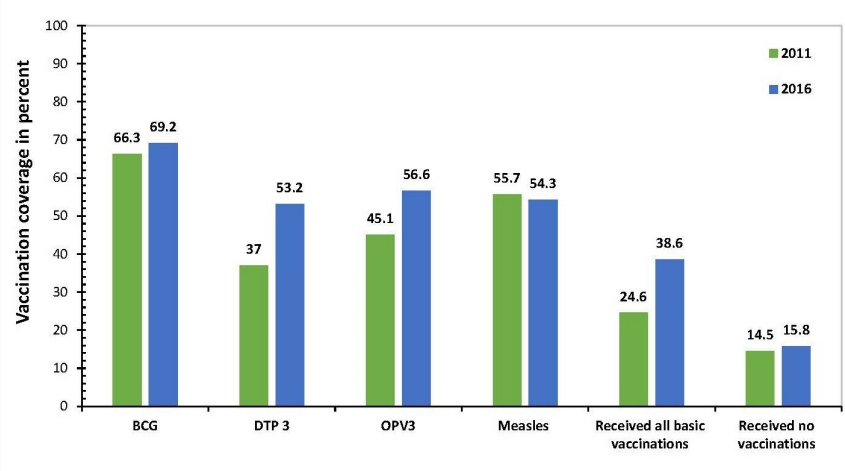

Figure 1 Vaccination coverage among children aged 12-23 months in Ethiopia (DHS 2011, 2016). BCG, Bacille Calmette-Guerin; DHS, Demographic and Health Survey; DTP diphtheria, tetanus toxoids and pertussis; OPV, oral polio vaccine.

The urban/rural differential in basic vaccination coverage increased from almost 28\% in 2011 to more than $29 \%$ in 2016 (table 2). Coverage remained low but showed slight increases from 2011 to 2016 in regions such as Afar $(8.5 \%-15.2 \%)$, Somali $(17.1 \%-21.8 \%)$ and Oromia $(15.6 \%-24.7 \%)$. Basic vaccination coverage also showed disparities between and within the regions of Ethiopia (table 2). For example, in 2016, coverage was $89.2 \%$ (95\% CI $82.0 \%$ to $93.8 \%$ ) and $67.3 \%$ (95\% CI $57.6 \%$ to $75.7 \%$ ) among children living in Addis Ababa and Tigray regions, respectively, whereas in Afar it was $15.2 \%$ (95\% CI $8.0 \%$ to $26.9 \%$ ) and Somali $21.8 \%$ (95\% CI $13.8 \%$ to $32.7 \%)$.

\section{Inequalities in vaccination coverage}

Inequalities in child vaccination persisted during 2011 and 2016: basic vaccination status was favourably concentrated among children from wealthier households while the distribution of those who received no vaccination remained disproportionately concentrated among poor (figure 2). The uptake of BCG, DTP3, OPV3, measles and basic vaccination were disproportionately concentrated among children from wealthy households during 2011 and 2016 (figure 3). DTP3 and basic vaccination status had lower coverage and

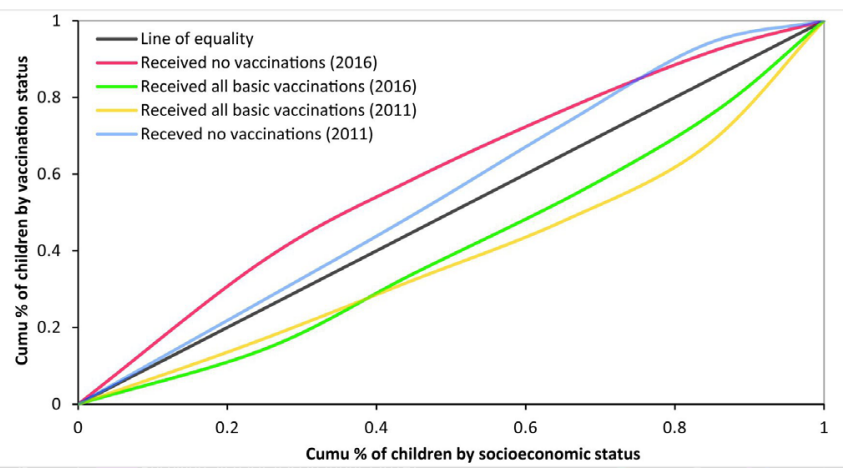

Figure 2 Concentration curves for child vaccination status, Ethiopia (DHS 2011, 2016). DHS, Demographic and Health Survey.

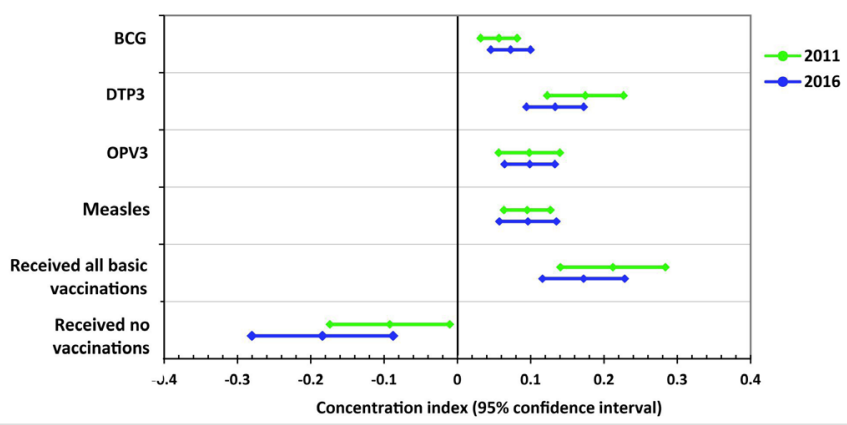

Figure 3 Concentration indexes that shows socioeconomic inequalities in child vaccinations, Ethiopia (DHS 2011, 2016). BCG, Bacille Calmette-Guerin; DHS, Demographic and Health Survey; DTP, diphtheria, tetanus toxoids and pertussis; OPV, oral polio vaccine.

showed the highest inequalities during 2011 and 2016; for example, in 2016, DTP3 had concentration index of $(\mathrm{CCI}=0.175)$ and basic vaccination $(\mathrm{CCI}=0.172)$. The estimate for the distribution of children who received no vaccination in 2011 was (CCI $=-0.092)$, this increased to $(\mathrm{CCI}=-0.184)$ in 2016 (figure 3$)$. The negative values for children who received no vaccination confirms pro-poor distributions. Increased vaccination coverage decreased inequalities as vaccinations such as BCG, OPV3 and measles that had higher coverage showed lower inequalities (figure 3 ).

The decomposition results in (table 3 ) and (figure 4) show that the significant contributors to socioeconomic inequality in basic vaccination status included, wealth, maternal education, contraceptive use, antenatal care contacts, exposure to media that include radio and TV, and place of residence (rural).

The decomposition analysis showed similar patterns in factors that explain socioeconomic inequalities in child vaccination status on both surveys. The use of maternal health services had the highest significant contributions to socioeconomic inequalities in child vaccination. Antenatal care contacts had $45.4 \%$ contribution in 2011 and $50.4 \%$ in 2016 . Wealth status is the other significant contributor, $23.9 \%$ in 2011 and $21.2 \%$ in 2016. On the other hand, rural residence had a negative contribution to socioeconomic inequalities in child vaccination on both surveys.

The overall concentration index for basic child vaccination was positive. Any significant positive contributor in (table 3) and (figure 4) means that socioeconomic inequality in basic vaccination would have been less prorich if: (1) the contributing variables (eg, antenatal care contacts or wealth) were to be evenly distributed among the rich and poor. Negative contributing variables (eg, rural residence) would cause the opposite effect. The residual or unexplained contributing factors to socioeconomic inequalities in basic vaccination account for $34.5 \%$ in 2011 and $-12 \%$ in 2016. 


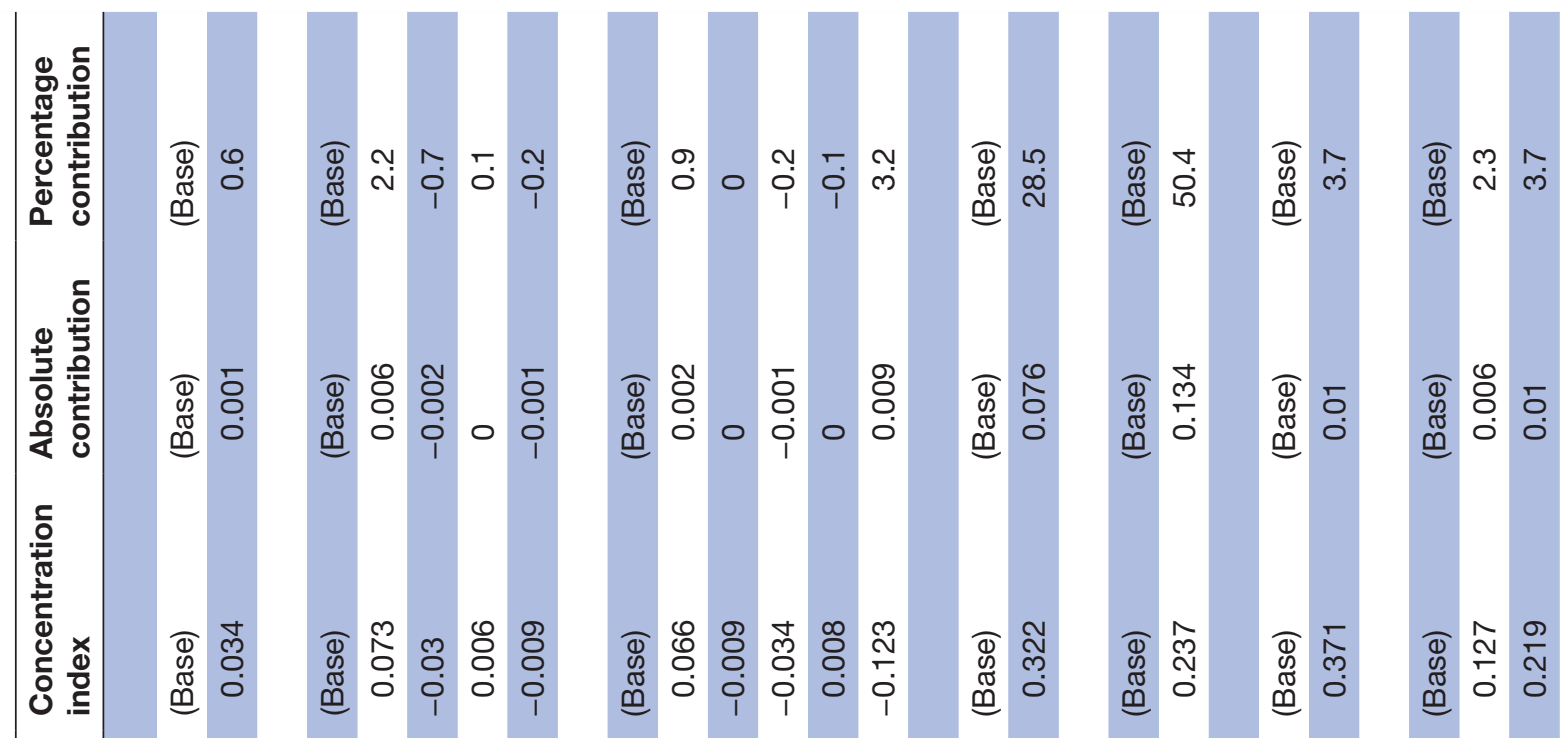

赵

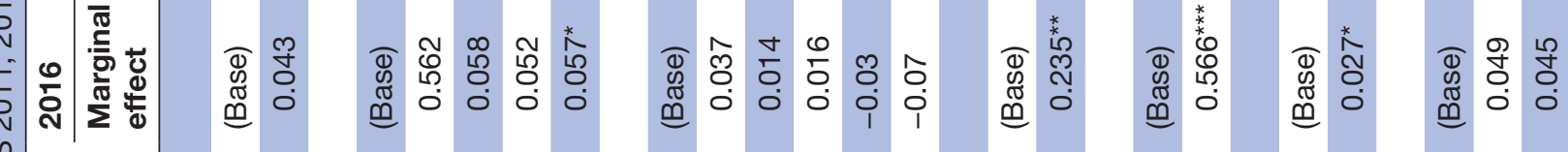

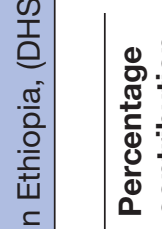

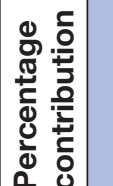

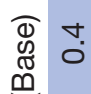

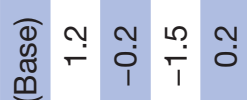

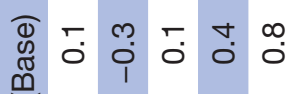

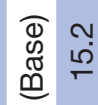

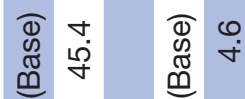

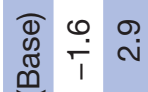

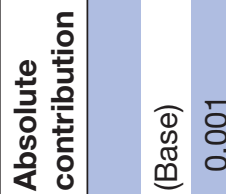

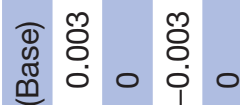

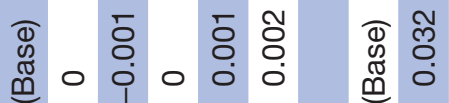

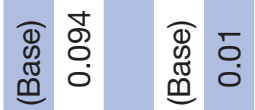

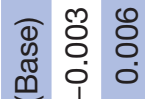

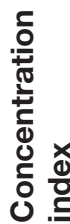

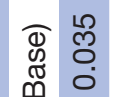

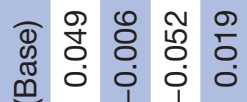

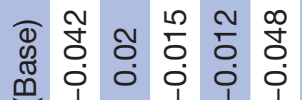

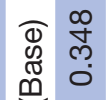

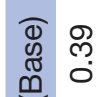

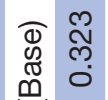

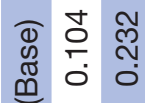

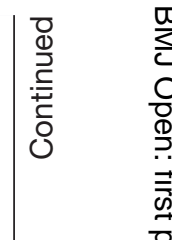

할

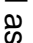

$\vec{\circ}$

$\overrightarrow{\vec{\omega}}$

홍.

กิ

N

फ्छ

$\vec{v}$

ก

\begin{tabular}{l}
0 \\
Oे \\
$\stackrel{0}{0}$ \\
$\stackrel{0}{0}$ \\
\hline
\end{tabular}

N

ํํㅇ

흥

$\frac{0}{8}$

훙

룽

흠

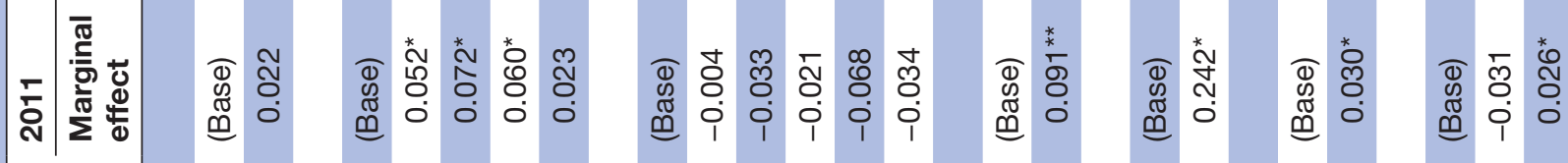
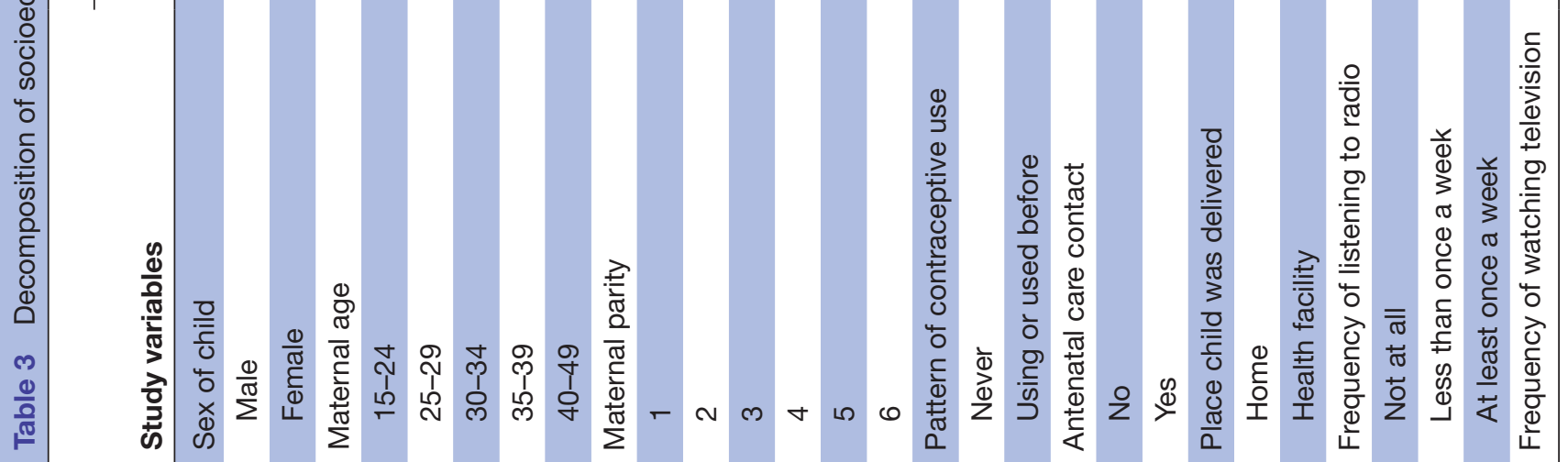

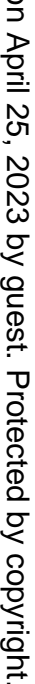




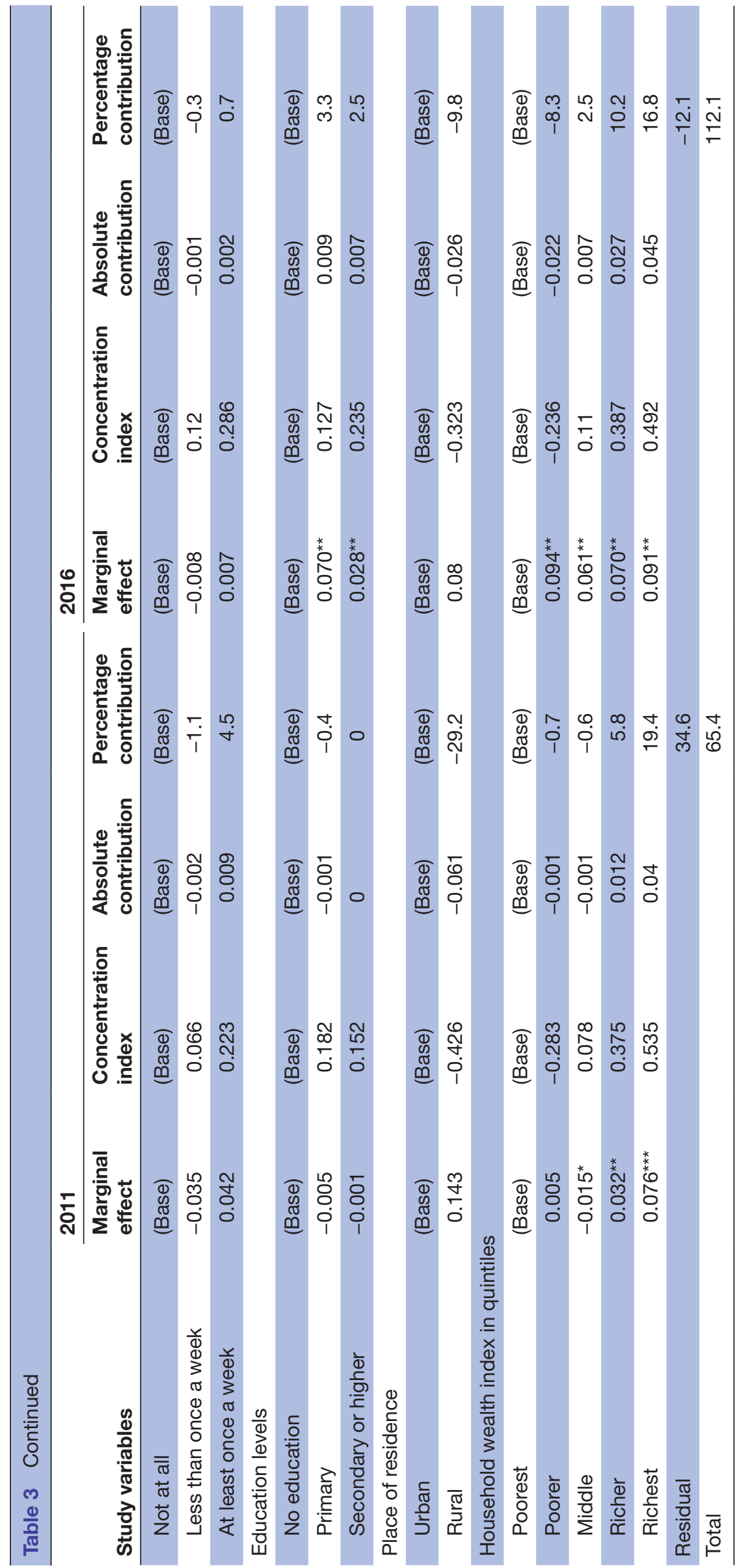

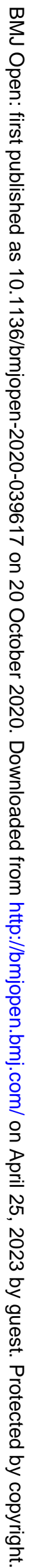




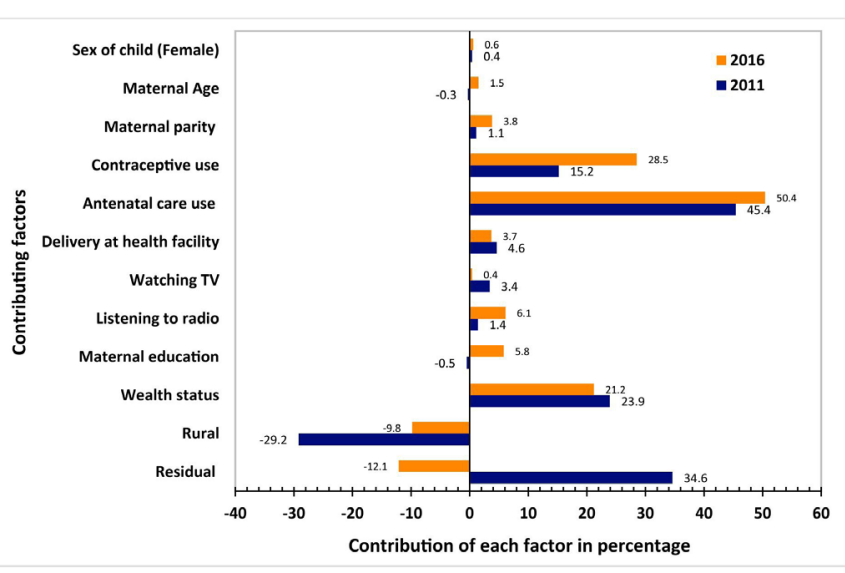

Figure 4 Percentage contributions of factors explaining socioeconomic inequalities in full vaccination coverage, Ethiopia (DHS 2011, 2016). DHS, Demographic and Health Survey; TV, television.

\section{DISCUSSION}

This study examined inequalities in vaccination coverage among children aged 12-23 months in Ethiopia. The uptake of all basic vaccinations increased from $24.6 \%$ in 2011 to $38.6 \%$ in 2016. Coverage improvements from 2011 to 2016 were observed in BCG vaccine uptake by $3 \%$, DTP3 by $16.2 \%$, OPV3 by $11.5 \%$ and all basic vaccinations by $14 \%$. While there were improvements in vaccination coverage from 2011 to 2016, issues that need to be addressed include a lack of awareness about vaccination, facilities' limited operating hours, maternal time constraints and the distance to facilities. ${ }^{34} 35$

Ethiopia remains one of the top 10 high priority countries in the world where children remain unvaccinated. ${ }^{1}$ In the current study, more than $15 \%$ of children received none of the vaccines, while incomplete vaccinations were $62 \%$ in 2011 and $46 \%$ in 2016. Possible explanations for under vaccination or no vaccination may include vaccine hesitancy or refusal, lack of access to vaccination services or missed opportunities for vaccination. ${ }^{5}$ Vaccine hesitancy refers to concerns about real or perceived vaccine adverse events among parents that may lead to delayed vaccination schedules or refusal of vaccinations altogether. ${ }^{36}$ Missed opportunities present another explanation in which children may not receive one or all of recommended vaccines even if they are vaccine eligible and can attend health facilities, which is commonly refers to missed opportunities. ${ }^{536}$

The uptake of BCG, DTP3, OPV3, measles and receipt of all basic vaccinations were disproportionately concentrated among children from wealthier households. This finding is consistent with multicountry studies across low-middle income countries. ${ }^{937}$ However, a study across three countries $^{14}$ that include Gambia, Kyrgyz Republic and Namibia showed that receipt of all basic vaccinations was disproportionately concentrated among children from poor households. The coverage of DTP3 and basic vaccination showed the highest inequalities favouring children from wealthy households. More than $14 \%$ of children received none of the vaccines during 2011 and
2016. These children were mainly from disadvantaged households; for example, children who remained unvaccinated in 2016 were $8.3 \%$ among the richest quantile, while $24 \%$ were from the poorest quantile. Basic vaccination coverage also showed significant variations across regions of the country; this ranged from $15 \%$ in Afar, and $21 \%$ in Somali, to $67.3 \%$ in Tigray and $89.2 \%$ in Addis Ababa. Afar and Somali regions are predominantly nomadic pastoralist areas, with relatively weaker health systems compared with Tigray, and Addis Ababa that have improved healthcare coverage. ${ }^{38}$

Ensuring access to all recommended vaccines for all children, regardless of sociodemographic or socioeconomic status, saves more lives and facilitates progress towards achieving Sustainable Development Goals (SDGs). ${ }^{39}$ SDGs target for child mortality aims to reduce neonatal mortality to lower than 12 deaths per 1000 live births and under- 5 mortality to lower than 25 deaths per 1000 live births across all countries. ${ }^{39}$ In 2016, the infant mortality rate was 48 deaths per 1000 live births, and the under-5 mortality rate was 67 deaths per 1000 live births in Ethiopia. ${ }^{23}$

Findings of the current study revealed that the use of maternal health services, maternal education, exposure to media and wealth had positive contributions to basic vaccination uptake. These findings align with other similar studies. ${ }^{111540}$ Maternal knowledge about vaccinations is a determinant for vaccination status, which may be associated with increased knowledge about benefits of child vaccination because of counselling during family planning and antenatal care contacts. ${ }^{41-43}$ Moreover, based on findings related to the impact of residence and access to health facilities on vaccination, it may be that a mother with regular access to family planning and antenatal care is also more likely to seek out postnatal care where vaccination of her child can be more readily provided. ${ }^{44}$

In the present study, maternal education had contributions to vaccine uptake in 2016, but this was not the case in 2011. Education helps to create improved awareness and knowledge about childhood vaccination. ${ }^{42}$ Previous studies have also indicated that educated women are more likely to take their child for vaccination. ${ }^{13} 42$ Exposure to media can also be a useful tool to reach population at different socioeconomic levels. The findings of this study showed that access to mass media (radio and TV) favourably influences vaccine uptake. Transmitting information about the importance of childhood vaccination is vital to reach not only mothers but also their partners and community leaders. ${ }^{45}$ Information dissemination that targets mother's partner and community leaders can help to create a conducive environment that can favourably influence mothers to vaccinate their children.

In the present study, the socioeconomic well-being of mothers was associated with higher vaccination uptake. While vaccinations are provided free of charge in many developing countries, mothers sometimes incur indirect costs, for example, transportation costs, which often limit their uptake of these services. ${ }^{6}$ Mothers at the lower 
wealth categories are more likely to experience challenges in accessing healthcare facilities as such less likely to take their child for vaccination. ${ }^{11}$

Children from rural areas had lower vaccination coverage compared with children from urban areas. In 2011, basic vaccination coverage was $48.2 \%$ in urban areas while it was $20.6 \%$ in rural areas. This pattern continued in 2016 , as $64.6 \%$ of children from urban areas had basic vaccination, but only $35.2 \%$ in rural areas. This finding is consistent with those of similar studies. ${ }^{11}{ }^{46}$ This could partly be explained by challenges faced in rural areas due to less developed health infrastructure and fewer skilled providers. ${ }^{47}$ In rural areas, long-distance to health facilities is another reason for low basic vaccination coverage. People live far from health facilities and the long distance, and lack of transportation poses a critical challenge for mothers to take their child for vaccination. ${ }^{47}$ Vaccines require cold chain management as it is sensitive to high temperatures. ${ }^{48}$ Health facilities in rural areas face a shortage of electric power supply to keep the cold chain equipment working, which could lead to cancellation of services as lack of cold chain equipment may result in the stock-out of vaccines. ${ }^{15}$ One study from Nigeria found that $47 \%$ of solar fridges for vaccine storage in eight states were broken. ${ }^{49}$

The strengths of this study include the use of a nationally representative survey from the two most recent DHS surveys. The decomposition of the contributing factors that drive socioeconomic inequalities in vaccination status provided a rich set of analysis for policy interventions to address socioeconomic disparities in access to basic vaccinations in Ethiopia. Limitations of the current study may include recall bias related to vaccination status as not all children had vaccination cards, and measures had to depend on the mother's verbal report. The DHS is a cross-sectional survey; it was not possible to establish temporality between childhood vaccination and explanatory factors.

\section{CONCLUSIONS}

The uptake of basic vaccination improved by $14 \%$ from 2011 to 2016, but the overall coverage remained low. Increased vaccine coverage was disproportionately concentrated among children from wealthy households, while the majority of children who had no vaccination were from disadvantaged households. Utilisation of reproductive and maternal health services, household income status and maternal education had significant positive contributions to improved vaccination status. Therefore, continued efforts at improving coverage of family planning, antenatal care contacts, institutional delivery, maternal education and socioeconomic well-being are required to improve vaccination status. Moreover, regions such as Afar, Somali, and Oromia, and rural areas of the country at large require targeting.
Acknowledgements Authors are grateful to ICF International for implementing the MEASURE DHS and making the data available for public use. United States Agency for International Development (USAID) funded the MEASURE DHS project.

Contributors FTB designed the study, analysed the data and drafted the manuscript. AH helped revise the study design and supervised the data analysis. All authors read and approved the final manuscript.

Funding The authors have not declared a specific grant for this research from any funding agency in the public, commercial or not-for-profit sectors.

\section{Competing interests None declared.}

Patient consent for publication Not required.

Ethics approval Ethics approval was not required for the current analysis, as the data were from publicly available DHS data. The MEASURE DHS /ICF International, Rockville, Maryland, USA — granted access to data on request.

Provenance and peer review Not commissioned; externally peer reviewed.

Data availability statement Data are available in a public, open access repository. The dataset was made available for public use after deidentification (data are available online at: https://www.idhsdata.org/idhs/)

Open access This is an open access article distributed in accordance with the Creative Commons Attribution Non Commercial (CC BY-NC 4.0) license, which permits others to distribute, remix, adapt, build upon this work non-commercially, and license their derivative works on different terms, provided the original work is properly cited, appropriate credit is given, any changes made indicated, and the use is non-commercial. See: http://creativecommons.org/licenses/by-nc/4.0/.

ORCID iD

Firew Tekle Bobo http://orcid.org/0000-0002-0960-1000

\section{REFERENCES}

1 World Health Organization. Immunization coverage, 2019. Available: https://www.who.int/news-room/fact-sheets/detail/immunizationcoverage [Accessed 19 Jan 2019].

2 Arsenault $\mathrm{C}$, Harper S, Nandi A, et al. Monitoring equity in vaccination coverage: a systematic analysis of demographic and health surveys from 45 Gavi-supported countries. Vaccine 2017;35:951-9.

3 Arsenault $\mathrm{C}$, Harper S, Nandi A, et al. An equity dashboard to monitor vaccination coverage. Bull World Health Organ 2017;95:128-34.

4 Federal Ministry of Health. A Ethiopia national expanded programme on immunization, 2015.

5 Porth JM, Wagner AL, Teklie $\mathrm{H}$, et al. Vaccine non-receipt and refusal in Ethiopia: the expanded program on immunization coverage survey, 2012. Vaccine 2019;37:2106-21.

6 World Health Organization. State of inequality: reproductive maternal newborn and child health: interactive visualization of health data. World Health Organization, 2015.

7 Victora CG, Requejo JH, Barros AJD, et al. Countdown to 2015: a decade of tracking progress for maternal, newborn, and child survival. The Lancet 2016;387:2049-59.

8 Arsenault C, Johri M, Nandi A, et al. Country-level predictors of vaccination coverage and inequalities in Gavi-supported countries. Vaccine 2017;35:2479-88.

9 Hosseinpoor AR, Bergen N, Schlotheuber A, et al. State of inequality in diphtheria-tetanus-pertussis immunisation coverage in low-income and middle-income countries: a multicountry study of household health surveys. Lancet Glob Health 2016;4:e617-26.

10 Shrivastwa N, Gillespie BW, Kolenic GE, et al. Predictors of vaccination in India for children aged 12-36 months. Am J Prev Med 2015;49:S435-44.

11 Ataguba JE, Ojo KO, Ichoku HE. Explaining socio-economic inequalities in immunization coverage in Nigeria. Health Policy Plan 2016;31:1212-24.

12 Branco FLCC, Pereira TM, Delfino BM, et al. Socioeconomic inequalities are still a barrier to full child vaccine coverage in the Brazilian Amazon: a cross-sectional study in Assis Brasil, ACRE, Brazil. Int J Equity Health 2014;13:118.

13 Acharya K, Paudel YR, Dharel D. The trend of full vaccination coverage in infants and inequalities by wealth quintile and maternal education: analysis from four recent demographic and health surveys in Nepal. BMC Public Health 2019;19:1673.

14 Hajizadeh M. Decomposing socioeconomic inequality in child vaccination in the Gambia, the Kyrgyz Republic and Namibia. Vaccine 2019;37:6609-16. 
15 McGavin ZA, Wagner AL, Carlson BF, et al. Childhood full and undervaccination in Nigeria, 2013. Vaccine 2018;36:7294-9.

16 Sachs JD. From millennium development goals to sustainable development goals. Lancet 2012;379:2206-11.

17 Tangcharoensathien V, Mills A, Palu T. Accelerating health equity: the key role of universal health coverage in the sustainable development goals. BMC Med 2015;13:101.

18 Ethiopian Public Health Institute (Ethiopia) and ICF. Ethiopia mini demographic and health survey 2019: key indicators. Rockville, Maryland, USA: EPHI and ICF, 2019.

19 World Health Organization. Regional strategic plan for immunization 2014-2020. World Health Organization. Regional office for Africa, 2015.

20 Mekonnen AG, Bayleyegn AD, Ayele ET. Immunization coverage of 12-23 months old children and its associated factors in MinjarShenkora district, Ethiopia: a community-based study. BMC Pediatr 2019;19:198.

21 Tamirat KS, Sisay MM. Full immunization coverage and its associated factors among children aged 12-23 months in Ethiopia: further analysis from the 2016 Ethiopia demographic and health survey. BMC Public Health 2019;19:1019.

22 Wondimu A, Cao Q, Wilschut JC, et al. Factors associated with the uptake of newly introduced childhood vaccinations in Ethiopia: the cases of rotavirus and pneumococcal conjugate vaccines. BMC Public Health 2019;19:1656.

23 Central Statistical Agency (CSA) [Ethiopia], ICF. Ethiopia demographic and health survey 2016. Addis Ababa, Ethiopia, and Rockville, Maryland, USA: CSA and ICF, 2016.

24 The DHS Program. The DHS overview. Available: https://dhsprogram. com/What-We-Do/Survey-Types/DHS.cfm

25 The DHS Program. DHS methodology. Available: https://dhsprogram. com/What-We-Do/Survey-Types/DHS-Methodology.cfm

26 The DHS Program. DHS questionnaires. Available: https:// dhsprogram.com/What-We-Do/Survey-Types/DHS-Questionnaires. $\mathrm{cfm}$

27 Rutstein SO, Rojas G. Guide to DHS statistics. 38. Calverton, MD: ORC Macro, 2006.

28 Restrepo-Méndez MC, Barros AJ, Wong KL, et al. Inequalities in full immunization coverage: trends in low- and middle-income countries. Bull World Health Organ 2016;94:794-805.

29 Solar O, Irwin A. A conceptual framework for action on the social determinants of health, 2010

$30 \mathrm{Kc} \mathrm{A}$, Nelin V, Raaijmakers $\mathrm{H}$, et al. Increased immunization coverage addresses the equity gap in Nepal. Bull World Health Organ 2017;95:261-9.

31 Doherty E, Walsh B, O'Neill C. Decomposing socioeconomic inequality in child vaccination: results from Ireland. Vaccine 2014;32:3438-44.

32 O'Donnell O, Van Doorslaer E, Wagstaff A, et al. Analyzing health equity using household survey data: a guide to techniques and their implementation: The world bank, 2007.
33 Wagstaff A, vE D, Watanabe N. On decomposing the causes of health sector inequalities with an application to malnutrition inequalities in Vietnam: The world bank, 2001.

34 Legesse E, Dechasa W. An assessment of child immunization coverage and its determinants in Sinana district, Southeast Ethiopia. BMC Pediatr 2015;15:31.

35 Tadesse H, Deribew A, Woldie M. Explorative assessment of factors affecting child immunization in Wonago district, Gedeo zone, South Ethiopia. Archives of Medical Science 2009;5:233.

36 Sadaf A, Richards JL, Glanz J, et al. A systematic review of interventions for reducing parental vaccine refusal and vaccine hesitancy. Vaccine 2013;31:4293-304.

37 Hajizadeh M. Socioeconomic inequalities in child vaccination in low/middle-income countries: what accounts for the differences? J Epidemiol Community Health 2018;72:719-25.

38 Federal Democratic Republic of Ethiopia [Ministry of Health]. Health sector transformation plan (HSTP) 2015/16-2019/20 (2008-2012 EFY). Federal Democratic Republic of Ethiopia Ministry of Health 2015.

39 United Nations. Transforming our world: the 2030 agenda for sustainable development. resolution A/RES/70/1. adopted September, 2015. Geneva: United Nations, 2015.

40 Mbengue MAS, Sarr M, Faye A, et al. Determinants of complete immunization among senegalese children aged 12-23 months: evidence from the demographic and health survey. BMC Public Health 2017;17:630.

41 Bhutta ZA, Das JK, Bahl R, et al. Can available interventions end preventable deaths in mothers, newborn babies, and stillbirths, and at what cost? Lancet 2014;384:347-70.

42 Burroway R, Hargrove A. Education is the antidote: Individualand community-level effects of maternal education on child immunizations in Nigeria. Soc Sci Med 2018;213:63-71.

43 Bobo FT, Yesuf EA, Woldie M. Inequities in utilization of reproductive and maternal health services in Ethiopia. Int $J$ Equity Health 2017;16:105.

44 Kerber KJ, de Graft-Johnson JE, Bhutta ZA, et al. Continuum of care for maternal, newborn, and child health: from slogan to service delivery. Lancet 2007;370:1358-69.

45 Zamawe COF, Banda M, Dube AN. The impact of a community driven mass media campaign on the utilisation of maternal health care services in rural Malawi. BMC Pregnancy Childbirth 2016;16:21.

46 Raza O, Lodhi FS, Morasae EK, et al. Differential achievements in childhood immunization across geographical regions of Pakistan: analysis of wealth-related inequality. Int J Equity Health 2018;17:122.

47 World Health Organization. State of inequality: childhood immunization, 2016.

48 Kartoglu U, Milstien J. Tools and approaches to ensure quality of vaccines throughout the cold chain. Expert Rev Vaccines 2014;13:843-54.

49 Ophori EA, Tula MY, Azih AV, et al. Current trends of immunization in Nigeria: prospect and challenges. Trop Med Health 2014;42:67-75. 